\title{
Assessment of diagnostic value of neutrophil to lymphocyte ratio and platelet to lymphocyte ratio as inflammatory indicators and indirect endothelial dysfunction markers in patients with acute pulmonary embolism
}

Paweł Obierzyński' ${ }^{1}$ Piotr Piech ${ }^{2,3}$, Grzegorz Staśkiewicz ${ }^{2,4}$, Gabriela Kuroska ${ }^{1,5}$, Maciej Kozioł², Lila Dąbkowska ${ }^{6}$

${ }^{1}$ Human Anatomy Research Group, Department of Human Anatomy, Medical University of Lublin

2 Department of Human Anatomy, Medical University of Lublin

${ }^{3}$ Department of Orthopedics and Traumatology, Medical University of Lublin

${ }^{4}$ 1st Department of Radiology, Medical University of Lublin

${ }^{5}$ Department of Neurosurgery and Pediatric Neurosurgery, Medical University of Lublin

${ }^{6}$ Department of Pharmacology and Pharmacodynamics, Medical University of Lublin

Obierzyński P., Piech P, Staśkiewicz G, Kuroska G, Kozioł G, Dąbkowska L. Assessment of diagnostic value of neutrophil to lymphocyte ratio and platelet to lymphocyte ratio as inflammatory indicators and indirect endothelial dysfunction markers in patients with acute pulmonary embolism. J Pre-Clin Clin Res. 2018; 12(1): 26-29. doi: 10.26444/jpccr/85716

\begin{abstract}
Introduction. Acute pulmonary embolism (APE) is a serious cardiovascular disease associated with high mortality rates despite advanced therapeutic options and widely used antithrombotic prophylaxis. The variety of nonspecific symptoms and comorbidities result in APE not being properly diagnosed in many patients. Therefore, identifying new, easily accessible and cheap diagnostic markers for the disease is important. Studies conducted for the last decade have undoubtedly confirmed the role of inflammation and endothelial damage in pathogenesis of APE, and the elevated NLR and PLR values have been considered as a new markers of inflammation.

Materials and method. Computed tomography pulmonary angiography (CTPA) and routine blood tests were performed in all the patients, after which neutrophil to lymphocyte ratio (NLR) and platelet to lymphocyte ratio (PLR) were calculated, based on neutrophil, platelet and lymphocyte counts. Finally, statistical analysis of the results in groups with confirmed and ruled-out APE was performed.

Results. There were no statistically significant differences in the values of NLR and PLR between patients with confirmed and ruled-out APE. Chi-square and Mann-Whitney tests were used with $\mathrm{p} \leq 0.05$ considered significant.

Conclusions. According to the results of this study, it was not possible to demonstrate the usefulness of NLR and PLR in the diagnostics of APE. To the best of the authors' knowledge, this is the first study in the literature considering the role of NLR and PLR in the diagnostics of APE.
\end{abstract}

\section{Key words}

acute plumonary embolism, platelet to lymphocyte ratio, Neutrophil to lymphocyte ratio, inflammation, endothelial damage

\section{INTRODUCTION}

Acute pulmonary embolism (APE) is a severe cardiovascular disease which most commonly develops secondary to deep vein thrombosis (DVT) and is a significant cause of morbidity and mortality worldwide, particularly in patients included in the risk group $[1,2,3]$. The essence of this illness is partial or total obstruction of the pulmonary vascular bed as an effect of presence of a thrombus through the blood flopw to the pulmonary artery or its branches from peripheral veins. In the clinical course of APE, the most common symptoms are dyspnea. dry cough. chest pain. as well as tachypnea and tachycardia. However. as a matter of fact. many nonspecific signs can also occur. especially in elderly patients and those with comorbidities $[1,2]$. These symptoms may occur in many

Address for correspondence: Piotr Piech, Department of Human Anatomy, Medical University of Lublin, Jaczewskiego 4, 20-090 Lublin, Poland

E-mail: ppiotr.md@gmail.com

Received: 12 December 2017; accepted: 24 February 2018 more common diseases, such as pneumonia. exacerbation of COPD or cardiac arrhythmias. which is why almost $27 \%$ of patients visited general practitionerbefore hospitalization [4]. Moreover, the time from the onset of symptoms to admission to hospital was significantly longer among patients who had first visited a primary care physician [4]. Depending on the sources. post-mortem examinations show that up to $80 \%$ of APE cases which were the direct cause of death. had not been properly diagnosed $[2,3]$.

Identification of blood parameters which may improve the diagnostics of APE, especially in combination with already used CT-derived parameters and biochemical markers, could help improve the general health of the population. Effective therapeutic interventions and elimination of adverse outcomes of delayed diagnosis and treatment seem to be important, particularly in the context of the progressive aging process of the population and increasing numbers of surgical procedures performed $[2,3]$. 


\section{OBJECTIVE}

Considering the role of inflammation and endothelial damage in the pathogenesis of venous thromboembolism (VTE), the aim of the study was to investigate the potential value of NLR and PLR in the diagnostics of APE. Moreover, detailed analysis of available literature led to the conclusion that this is the first study to investigate the potential significance of these ratios in the context of the diagnostics of APE.

\section{MATERIALS AND METHOD}

The retrospective analysis included the clinical data of 160 patients hospitalized in the Department of Orthopedics and Traumatology of the Medical University in Lublin, eastern Poland. All of them were after large surgical procedures and/or were long-term immobilized during hospitalization. In all patients, because of the clinical suspicion of APE, computed tomography pulmonary angiography (CTPA) and routine blood tests were performed. Venous blood samples were taken from upper limbs veins within 24 hours since the occurrence of symptoms and collected in calcium EDTA tubes. Within 30 minutes after blood sampling, blood parameters were evaluated by automated count analyzer. In 72 patients, APE was confirmed based on CTPA results obtained by a standard protocol used in the Medical Uniuversity hospital; in 88 patients the PE was definitely ruled-out (Fig. 1).

The neutrophil to lymphocyte ratio (NLR) and platelet to lymphocyte ratio (PLR) values were retrospectively calculated in both groups, based on neutrophil. platelet and lymphocyte counts; statistical analysis of results obtained was performed with the SPSS 16.0 statistical package. Chi-square and Mann-Whitney tests were used with $\mathrm{p} \leq 0.05$ considered significant. Analyzed parameters are presented as minimal. maximal and median.

\section{RESULTS}

The patients were divided into two groups based on the CTPA results - Group A (72 patients) and Group B (88 patients) - with confirmed and ruled-out APE. respectively. The basic characteristics of the study groups are summarized in Table 1. There were no significant differences in gender. nor by age distribution between groups.

Table 1. Basic characteristic of study groups. Numbers are median. minimal and maximal value or percentages where indicated

\begin{tabular}{lccc}
\hline & $\begin{array}{c}\text { Group A } \\
(\mathrm{n}=72)\end{array}$ & $\begin{array}{c}\text { Group B } \\
(\mathrm{n}=88)\end{array}$ & $\mathbf{p}$ \\
\hline age [years] & $65.5(20-89)$ & $61.0(21-94)$ & 0.230 \\
\hline females & $48.61 \%$ & $53.41 \%$ & 0.546 \\
\hline
\end{tabular}

Table 2 presents the results of NLR and PLR calculations and statistical analysis. No significant differences in NLR nor PLR values were revealed between groups. Detailed distribution of NLR and PLR values in the study groups is presented in Figures 2 and 3, respectively.
Table 2. NLR and PLR values in study groups. Numbers are median. minimal and maximal value

\begin{tabular}{lccc}
\hline & $\begin{array}{c}\text { Group A } \\
(\mathrm{n}=72)\end{array}$ & $\begin{array}{c}\text { Group B } \\
(\mathrm{n}=88)\end{array}$ & $\mathbf{p}$ \\
\hline $\mathbf{N L R}$ & $5.28(1.03-43.19)$ & $4.83(0.04-44.13)$ & 0.631 \\
\hline PLR & $258.27(52.26-1396.15)$ & $224.45(8.64-726.67)$ & 0.112 \\
\hline
\end{tabular}

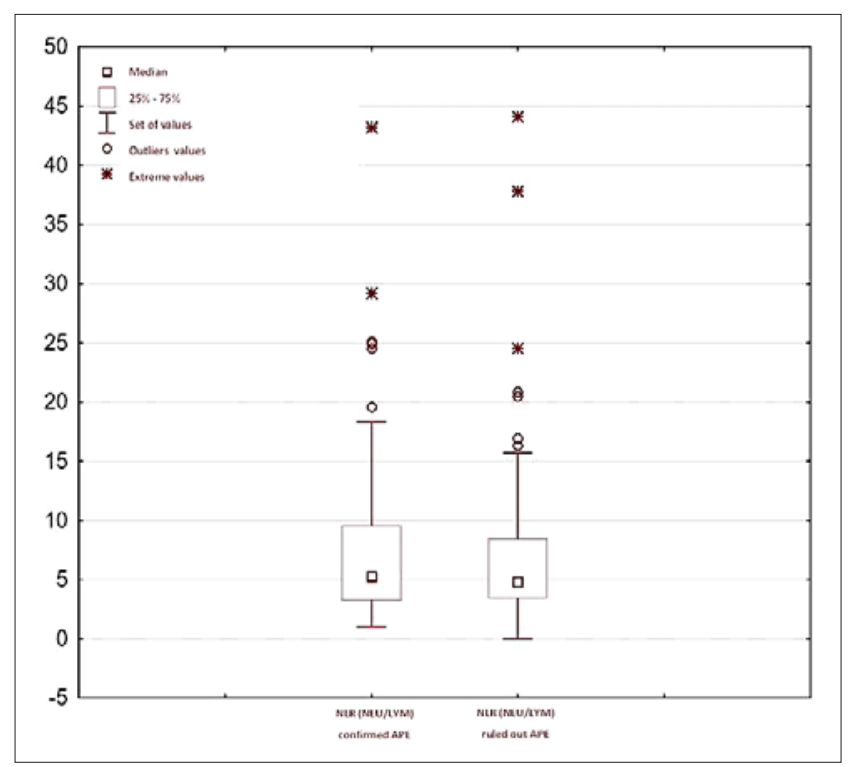

Figure 2. Distribution of NLR in both groups of patients

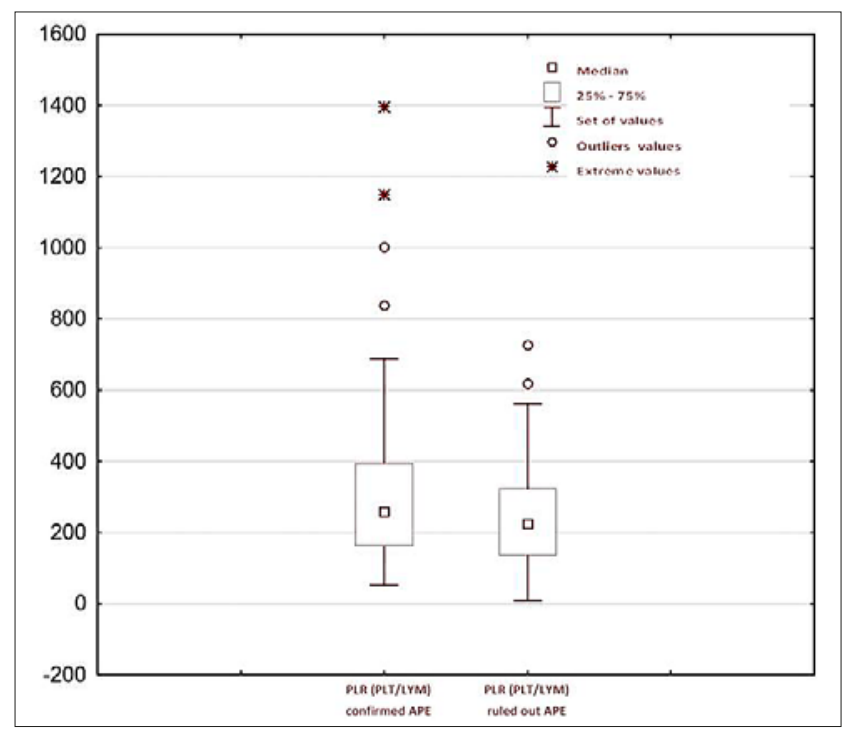

Figure 3. Distribution of PLR in both groups of patients

\section{DISCUSSION AND CONCLUSIONS}

Pulmonary embolism as a component of venous thromboembolism (VTE) is the third most common cardiovascular disease in Europe. Due to a variety of symptoms, insufficient availability of CTPA and unknown specific biochemical markers it still correlates with a high mortality rate despite widely used antithrombotic prophylaxis [1-4].

According to studies performed in recent years, the role of inflammation and endothelial damage in the pathogenesis of APE has been confirmed [5-8]. NLR reflects 
the balance between neutrophils and lymphocytes taking part in inflammatory response, and its elevated value is the result of an increased neutrophil count and decreased lymphocyte count $[7,8]$. It is suspected that the development of a thrombus in a blood vessel is initiated by inflammation within vessel wall in which granulocyteinflow (caused by chemoattractants releasing), their degranulation and reactive oxygen species (ROS) production, are direct causes of endothelial dysfunction [9-12]. In this way, procoagulants factors in the vessel wall are released, and then the activated platelets contribute to a thrombus formation and maintain its increase $[11,12,14]$. Regarding to neutrophils, their role is not limited to initiating coagulation - they are immobilized between platelets and, as an element of the thrombus, they can recruit other cells to take part in the coagulation cascade, especially in venous valves where the blood flow is minimal [7, 12-14]. Moreover, the neutrophils, as well as platelets, can inhibit natural anticoagulant pathways and reduce plasma fibrinolytic activity $[7,14]$ The elevated granulocyte count was seen to be associated with a much higher risk of thromboembolism in oncological patients [15]. Lymphopenia, frequently observed during inflammatory events, is caused by accelerated apoptosis which is secondary to elevated serum levels of corticosteroids released by suprarenal glands as a response to acute stress conditions, such as APE and acute coronary syndrome $[8,16,17]$.

It has also been demopnstrated that an increased level of cortisol stimulates the production of neutrophils in bone marrow; however, their accelerated releasing to blood correlates with immature morphology and incomplete immunological function $[8,16,17]$. The role of platelets in development of thrombus had been suspecting since Virchow and has been already confirmed, but recently an elevated PLR has been considered as a new marker of systemic inflammation. as well as platelet parameters, such as MPV, have been recognized as potentially useful in the diagnostic of APE $[18,19,20]$. Moreover. it was found that NLR and PLR values correlate together with endothelial dysfunction as better indicators of inflammation compared to WBC count, especially in combination with biochemical markers [21-24]. It is suspected. that the role of PLR value can be particularly valuable in clinical practice because it gives information about both the pathways of APE pathogenesis - it integrates the information about primary haemostasis and inflammation $[24,25]$. This seems to be more useful than platelet and lymphocyte counts alone $[21-24,26]$.

Based on the findings of the aforementioned studies, the authors hypothesize that PLR and NLR might be easily accessible and cheap indicators useful in the diagnostics of pulmonary embolism, which could play a role in daily clinical practice. Both NLR and PLR are commonly available in routinely performed laboratory tests in outpatient as well as inpatient care. The identification of new diagnostic factors, especially in combination with CT-derived and biochemical parameters, could help to shorten the time before admission to hospital, guide effective interventions and avoid adverse events of delayed treatment, even if the patient presents unobvious or nonspecific symptoms. However, according to the results of this study, it is not able possible to demonstrate the utility of NLR and PLR in diagnostics of APE - there were no statistically significant differences in the values of the mentioned parameters between patients with confirmed and rule- out pulmonary embolism.
On the other hand, it should be mentioned that the presented study was a single centre retrospective study with a relatively limited sample size. Considering this fact and the exploratory nature of the study, the authors maintain that the diagnostic values of NLR and PLR remain to be evaluated by further observations and studies which will undoubtedly be performed.

\section{REFERENCES}

1. Konstantinides SV, Torbicki A, Agnelli G, et al. ESC Committee for Practice Guidelines (CPG). Guidelines on the diagnosis and management of acute pulmonary embolism: the Task Force for the Diagnosis and Management of Acute Pulmonary Embolism of the European Society of Cardiology (ESC). EurHeart J 2014; 35: 3033-69.

2. Strzelecki Z, Szymborski J, et al. Zachorowalność i umieralność na choroby układu krążenia a sytuacja demograficzna Polski. Warsaw: Rządowa Rada Ludnościowa. 2015.

3. Cohen AT, Agnelli G, Anderson FA, Arcelus JI, Bergqvist D, Brecht JG, et al. (VTE) in Europe. The number of VTE events and associated morbidity and mortality. ThrombHaemost. 2007; 98: 756-64.

4. Życińska K, Wiktorowicz M, Tomasik D, et al. Clinical presentation of pulmonary embolism in general practice. Family Medicine \& Primary Care Review 2013; 15(3): 430-433.

5. Fox EA, Kahn SR. The relationship between inflammation and venous thrombosis. A systemic review of clinical studies. ThromHaemost 2005; 94: 362-5.

6.Zee RY, Glynn RJ, Cheng S, et al. An evaluation of candidate genes of inflammation and thrombosis in relation to the risk of venous thromboembolism: the women's genome health study. CircCardiovasc Genet. 2009; 2: 57-62.

7.Aksu K, Donmez A, Keser G. Inflammation-induced thrombosis: mechanisms. disease associations and management. Curr Pharm Des. 2012; 18: 1478-93.

8. Zahorec R. Ratio of neutrophil to lymphocyte counts-rapid and simple parameter of systemic inflammation and stress in critically ill. BratislLekListy. 2001; 102: 5-14.

9. Downing LJ, Strieter RM, Kadell AM, et al. Neutrophils are the initial cell type identified in deep venous thrombosis induced vein wall inflammation. Asaio J 1996; 42: 677-82.

10. Watts JA, Zagorski J, Gellar MA, et al. Cardiac inflammation contributes to right ventricular dysfunction following experimental pulmonary embolism in rats. J Mol Cell Cardiol. 2006; 41: 296-307.

11. Heestermans M, Salloum-Asfar S, Salvatori D, et al. Role of platelets. neutrophils. and factor XII in spontaneous venous thrombosis in mice. Blood. 2016; 127(21): 2630-2637. doi:10. 1182/ blood-2015-10-672766.

12. Pfeiler S, Stark K, Massberg S, Engelmann B. Propagation of thrombosis by neutrophils and extracellular nucleosome networks. Haematologica 2016; 102(2): 206-221. doi:10.3324/haematol.2016.142471

13. Kimball AS, Obi AT, Diaz JA, Henke PK. The emerging role of NETs in venous thrombosis and immunothrombosis. Front Immunol. 2016; 7(JUN): $1-8$.

14. Stewart GJ, Ritchie WG, Lynch PR. Venous endothelial damage produced by massive sticking and emigration of leukocytes. Am J Pathol. 1974; 74: 507-32.

15. Trujillo-Santos J, Di Micco P, Iannuzzo M, et al. Elevated white blood cell count and outcome in cancer patients with venous thromboembolism. Findings from the RIETE Registry. ThrombHaemost 2008; 100: 905-11.

16. Falk E, Shah PK, Fuster V. Coronary plaque disruption. Circulation 1995; 92: 657-71.

17. Hoffman M, Blum A, Baruch R, et al. Leukocytes and coronary heart disease. Atherosclerosis 2004; 172: 1-6.

18. Virchow R. Thrombose und Embolie. Gefässentzündung und septische Infektion. Gesammelte Abhandlungen zur wissenschaftlichen Medicin. Frankfurt am Main: Von Meidinger\&Sohn; 1856. p. 219-732.

19. Huang J, Chen Y, Cai Z, Chen P. Diagnostic value of platelet indexes for pulmonary embolism. Am J Emerg Med. 2015 Jun; 33(6): 760-3.

20.Piech P, Obierzyński P, Staśkiewicz G, et al. The analysis of selected morphotic parameters of blood as potential diagnostics factors in pulmonary embolisms. Journal of Education. Health and Sport 2017; 7(5): 458-469.

21. Kurtipek E, Buyukterzi Z, Buyukterzi M, Alpaydin MS, Erdem SS. Endothelial dysfunction in patients with pulmonary thromboembolism: 
neutrophil to lymphocyte ratio and platelet to lymphocyte ratio. ClinRespir J. 2015.

22. Balta S, Demirkol S, Kucuk U. The platelet lymphocyte ratio may be useful inflammatory indicator in clinical practice. Hemodial Int. 2013; 17: 668-9.

23. Afzal A, Noor HA, Gill SA, et al. Leukocytosis in acute pulmonary embolism. Chest 1999; 115: 1329-32.

24. Sunbul M, Gerin F, Durmus E, et al. Neutrophil to lymphocyte and platelet to lymphocyte ratio in patients with dipper versus nondipper hypertension. ClinExpHypertens. 2014; 36: 217-21.
25. Yüksel M, Yıldız A, Oylumlu M, et al. The association between platelet/lymphocyte ratio and coronary artery disease severity. AnadoluKardiyolDerg; 2014.

26. Buxhofer-Ausch V, Steurer M, Sormann S, et al. Influence of platelet and white blood cell counts on major thrombosis - analysis from a patient registry in essential thrombocythemia. Eur J Haematol. 2016; 97(6): 511-516. 